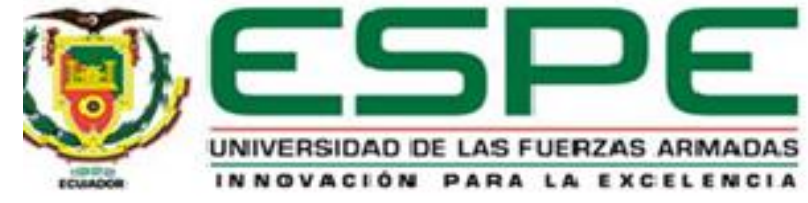

\title{
Augmented Reality System for the Assistance of Unmanned Aerial Vehicles
}

\author{
Misse Baldias, Eddy Santiago y Villacrés Vargas, Stalin Alfredo \\ Departamento de Eléctrica y Electrónica
}

Carrera de Ingeniería en Electrónica e Instrumentación

Artículo académico, previo a la obtención del título de Ingeniera en Electrónica e Instrumentación

Dr. Andaluz Ortiz, Víctor Hugo

05 de marzo del 2021 


\title{
Augmented Reality System for the Assistance of Unmanned Aerial Vehicles
}

\author{
Eddy S. Misse, Stalin A. Villacrés, Paola M. Velasco and \\ Víctor H. Andaluz \\ Universidad de las Fuerzas Armadas ESPE \\ Sangolquí Ecuador \\ \{esmisse, savillacres2, pmvelasco1, vhandaluz1\}@espe.edu.ec
}

\begin{abstract}
This article proposes the development of a virtual and augmented reality environment to assist in the assembly and maintenance of an unmanned aerial vehicle. Taking the lack of technological knowledge as the research gap, the study provides researchers and practitioners with new solutions through the familiarization and guided assistance of a UAV. The system is based on the user tracing to locate the position of the hands and validate in real-time if the actions performed are correct. In the interface can also access to virtualized environment so that the user define a route with the movement of his hands and immediately the UAV follow the path marked from the beginning to the end, the errors are displayed on the same screen to verify the stability of control.
\end{abstract}

Keywords - Tracking, UAV, virtualization, mixed reality, kinect.

\section{INTRODUCTION}

Virtual reality is currently used as a technique or method for human-machine communication because of the ease of being able to visualize environments designed in software [1]. An environment developed for applications in augmented reality allows real-time interaction with the virtual system, using $2 \mathrm{D}$ or $3 \mathrm{D}$ patterns as markers for the recognition of each object in the application despite the development of technology to include new forms of recognition such as motion sensors [2,3]. Communication-oriented virtual reality includes an avatar for each person and they can interact in the same environment without the need for people to be in only one part of the world $[4,5]$. Mixed reality comprises a more complex system, in this case the virtual world is superimposed on the real world, and requires feedback to capture any order or movement introduced by the user, is used in video games, in medicine and especially in engineering that aims to optimize resources, time and cost. [6]

Immersive technologies such as virtual reality (VR) and augmented reality (AR) are showing progress and adding new applications in industrial practices, engineering, marketing and others [7]. The implementation of immersive technologies in the industry leads to both technological and production growth, either through maintenance assistance or staff training. One of the main objectives of the immersive technology is to reinforce the theoretical knowledge of the workers using software developed for training in a specific industrial process [8]. To apply the software in engineering it is necessary to carry out predetermined tasks for the operator to perform in a set time or until the task is completed [9]. The aim is for the user to be in contact with this technology, to feel immersed in these realities in order to achieve established objectives in a more viable and dynamic way [10].

In the field of engineering, applications developed in an augmented reality simulation environment presents manufacturing processes, detect collisions between parts of a real workshop and a virtual product such as car bodies [11]. In industrial processes, the remote operation of robots reduces the danger for people because they operate remotely guided in an augmented reality environment [12]. Another application also exploited with augmented reality is the assembly of objects where its function is to give information of each step, virtual demonstrations, instructions and even show warning messages if necessary [13]. Image recognition optimizes augmented reality applications by providing object recognition in which an interactive menu can be mounted allowing the operator to manipulate and navigate between the options presented [14].

As described in the previous paragraphs, this work proposes the development of an augmented reality application that will be oriented to the assistance of assembly, maintenance and autonomous control of an unmanned aerial vehicle, in order to minimize errors when assembling the UAV, by means of feedback, the movements made by the operator are detected, who will visualize in real-time if the actions were corrects. Furthermore, with the implementation of a closed-loop control algorithm, it is intended to simulate the tracking of a path drawn by the user, which will be observed in the virtual environment.

There are different works related to robotics and industrial processes using augmented reality, which can be classified according to the type of interaction, including the following. $i$ ) Multimedia Interaction present multimedia information such as data sheets, audios and videos when scanning with the smartphone to the respective instrument or device as an aide to the user [3]. ii) Interaction with Animation: show information by means of movements or 3D animations of a process or device, in addition they show in detail the internal and external elements of the same one by means of the screen of the intelligent telephone [15]; iii) User interaction: there is real-time interaction with the application to recognize and follow the user's movements as an aid to the manipulation of the virtualized models in order to have a personalized training of some equipment to protect the integrity of the user or the instrument [16].

The article proposes an augmented reality application for assistance, maintenance and autonomous control processes in UAVs, using a kinect camera for real-time interaction with user 
movements, databases for image or 3D object recognition and programming codes to structure the different scenes, in order to ensure proper handling and operation of the UAVs.

\section{SYSTEM STRUCTURE}

A correct virtual assistance is not only about showing how to do a certain action, but in verifying if it is doing it right, that is to say, having a pattern that guides the operator to perform the correct action in a virtual environment. For this it is necessary to have the minimum quantity of errors and failures at the moment of tracking the operator, even if the control law developed is right there will exist visible errors and the correct assistance's objective towards the operator would lose sense, even with the appropriate tracking it is possible to establish routes or virtual paths that facilitate later assistances.

Nowadays, the autonomy of a UAV is essential, applications are continuously developed that follow paths defined by the same operator. A specific movement made by the user and interpreted as the desired route, so a control system is implemented to follow that route, display the errors and correct them if necessary or continue with the operation. And what better if the route can be visualized at that time using virtualized models of real environments, a great help for the operator is to indicate with his hand the route he wants his autonomous unmanned vehicle to follow, it is added that the UAV must be implemented with a control that allows me to realize the route marked by the operator without any problem.

This article proposes a system in augmented reality oriented to the assistance in maintenance processes, assembly as the virtualized placement of camera and propellers in addition to the control of unmanned aerial vehicles, through the identification of the upper extremities of the user and the simulation of the operation in 3D. Addition the training for the handling of new equipment is indispensable if it is required to perform at its maximum capacity from the beginning, the development of the virtualized environments focuses on the easy access to the assistance emulating a real physically environment, due to the feedback of the system corrects any failure made by the person in charge while operating the equipment.
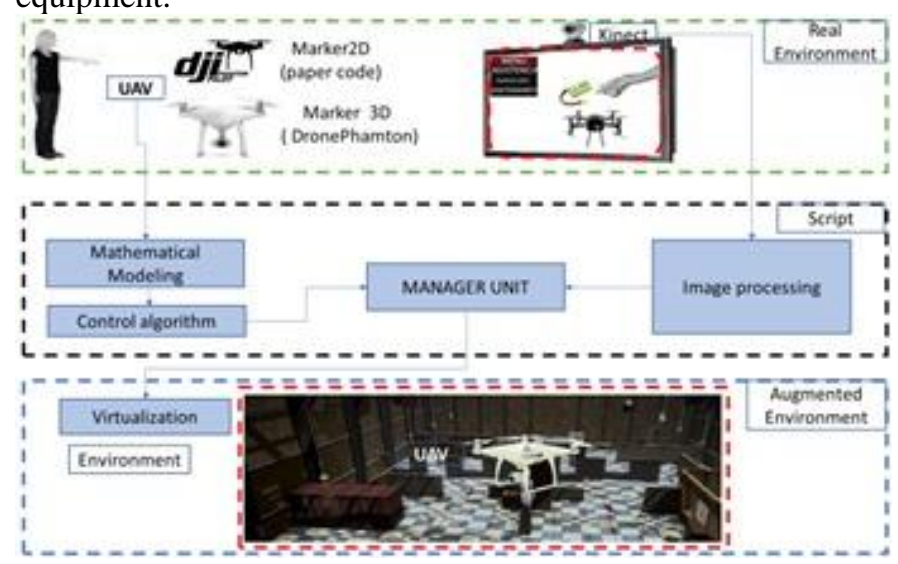

Figure 1. Structure proposed system

The proposed scheme for the application of the system is shown in Fig.1. The system consists of a Real environment, the programming of SCRIPTS that are processed in the same manager unit, Augmented Environment that interacts directly with the user's gestures.

The Real Environment at this stage there is the interaction of the user with the type of marker 2D (Paper Code) and 3D (Drone Phantom) that detects the camera, the application is displayed on the monitor in order to introduce the user to the virtual environment and allow him to interact by means of structured gestures.

The Script with 4 main blocks in order to develop: a) Image processing: The Kinect sensor is used to recognize the user's movements and take their reference points in space $(\mathrm{x}, \mathrm{y}, \mathrm{z})$, for later comparison and scaling in the Manager Unit; $b$ ) Mathematical Modeling: A mathematical model is developed based on the dynamic characteristics of the UAV (phantom 4); c) Control Algorithm: A closed-loop control algorithm for the UAV is implemented to perform autonomous, user-defined navigation tasks; d) Manager Unit: By means of software suitable for processing the acquired data and introducing them into a complete augmented reality system.

The Augmented Environment: It shows an interface for the user to interact in real time with the virtual objects and carry out the tasks of assembly, maintenance and autonomous navigation of the unmanned aerial vehicle.

\section{VIRTUAL ENVIRONMENT}

For the development and operation of the proposed application, three stages are considered, as shown in Fig. 2. These stages include: i) upper limb tracking for interaction with the environment $i$ ) virtual (2D) and real (3D) assembly of the UAV iii) autonomous navigation of the UAV with user defined routes.

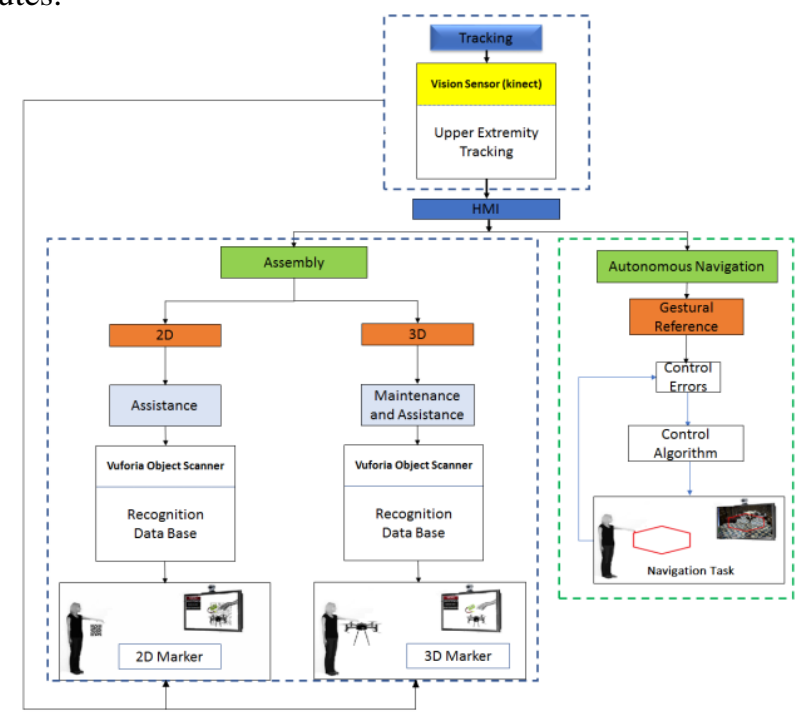

Figure 2. Diagram operation of application

Tracking: At this stage, the kinect sensor is used to identify and follow the characteristic points of the human operator's upper joints in order to virtualize them in a CAD model and thus allow the user to interact in real time with the application. It also recognizes hand gestures (open-closed) that allow the manipulation of virtual objects in the scenes. 
Assembly: The application was divided according to its function: a) Marker 2D (Paper Code): A database is created in Vuforia for the detection of images for the respective virtualized projection of the UAV (Phantom 4) and its HMI on the monitor, the user has the assistance to carry out the assembly tasks in a virtual drone; b) Marker 3D (Drone Phantom): The camera recognizes the real UAV (phantom 4) and then deploys an HMI. The user interacts with the real UAV and is assisted with the correct placement of the battery and propellers, as well as with the monitor feedback if the movements are incorrect.

Autonomous Navigation: The user generates with his hand a "Gesture Reference" trajectory that will be the desired path, with a closed loop control the UAV starts its autonomous flight in that trajectory observing in the monitor the relationship between the desired trajectory and the real one in order to obtain the existing control errors.

\section{AutOnOMOUS NAVIGATION}

For the control and navigation of the autonomous vehicle a closed-loop control diagram is proposed, see Fig.3. This Section is divided in 3 subsection: kinematic modeling of the UAV, control algorithm and stability analysis.

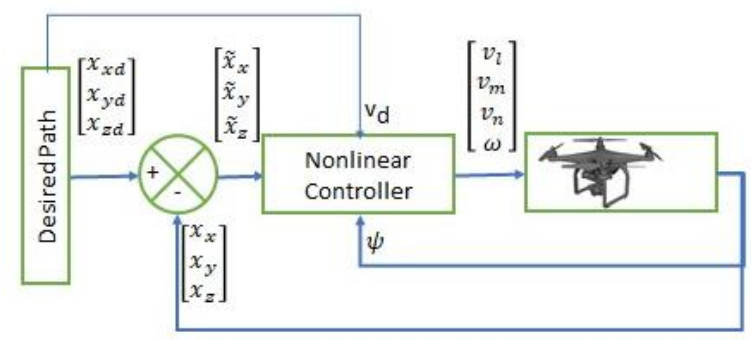

Figure 3. Closed-loop control diagram for the UAV

\section{A. Cinematic modeling of the UAV}

The modeling takes into account the characteristics and constraints of the UAV, representando $\left(v_{l}, v_{m}, v_{n}\right)$ las velocidades de maniobrabilidad del UAV sobre el sistema referencial $\langle R\rangle$ see Fig.4.

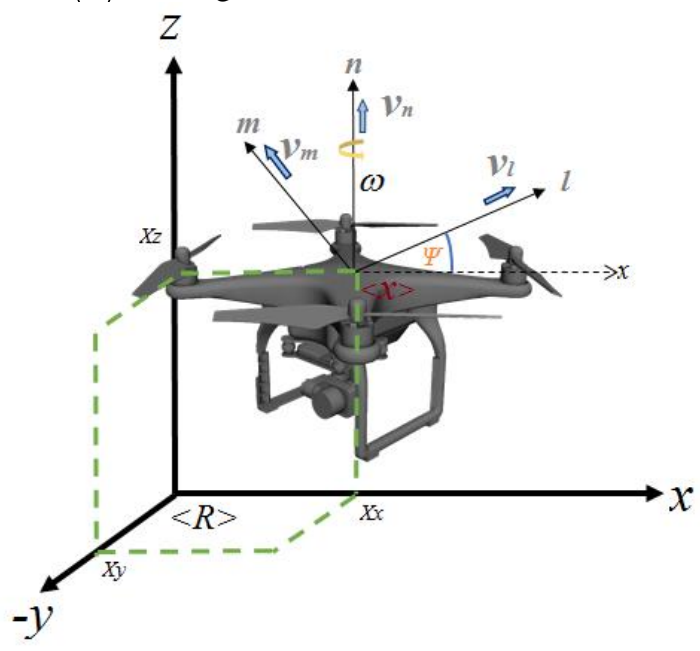

Figure 4. Kinematic scheme of UAV
Then the kinematic model of the UAV is equal to the linear velocities $x_{x}, x_{y}, x_{z}, \psi$ and are measured with respect to the reference plane $\langle R\rangle$, from where the following equations are obtained:

$$
\left\{\begin{array}{l}
\dot{x}_{x}=v_{l} \cos \psi-v_{m} \sin \psi \\
\dot{x}_{y}=v_{l} \sin \psi-v_{m} \cos \psi \\
\dot{x}_{z}=v_{n} \\
\psi=\omega
\end{array}\right.
$$

To represent the system of the model as a single equation proceeds to express in matrix form

$$
\begin{gathered}
{\left[\begin{array}{c}
\dot{x}_{x} \\
\dot{x}_{y} \\
\dot{x}_{z} \\
\psi
\end{array}\right]=\left[\begin{array}{cccc}
\cos \psi & -\sin \psi & 0 & 0 \\
\sin \psi & \cos \psi & 0 & 0 \\
0 & 0 & 1 & 0 \\
0 & 0 & 0 & 1
\end{array}\right]\left[\begin{array}{c}
v_{l} \\
v_{m} \\
v_{n} \\
\omega
\end{array}\right]} \\
\dot{\mathbf{x}}(t)=\mathbf{J}(\psi) \mathbf{v}(t)
\end{gathered}
$$

where, $\mathbf{J}(\psi)$ represents the Jacobian matrix that mapping between the velocities del $\operatorname{UAV} \mathbf{v}(t)$ and the vector of linear velocities $\dot{\mathbf{x}}(t)$.

\section{B. Control Algorithm}

Using numerical methods to find a control algorithm the (2) is defined at instant time $k$ to calculate the evolution of the system. The values of $\dot{\mathbf{x}}(t)$ in discrete time $t=k T_{0}$ are called $\mathbf{x}(k)(3)$. [17]

$$
\frac{(\mathbf{x}(k)-\mathbf{x}(k-1))}{T_{0}}=\mathbf{J}(\psi(k)) \mathbf{v}(k)
$$

where $T_{0}$ it represents the sampling time and $k \in\{1,2,3,4 \ldots\}$.

In order for the UAV to follow the path set by the user, the following expression is considered:

$$
\frac{1}{T_{0}}(\mathbf{x}(k)-\mathbf{x}(k-1))=\mathbf{v}_{\mathbf{d}}(k)+\frac{1}{T_{0}}\left(\mathbf{W}\left(\mathbf{P}_{\mathbf{d}}(k-1)-\mathbf{x}(k-1)\right)\right)
$$

where $\mathbf{P}_{\mathbf{d}}$ is the desired path, $\mathbf{W}(\tilde{\mathbf{x}}(k-1))$ is a diagonal matrix that control error weights, define as:

$$
\mathbf{W}\left(\tilde{\mathbf{x}}_{m}(k-1)\right)=\frac{\mathbf{W}_{m}}{1+|\tilde{\mathbf{x}}(k-1)|}
$$

where $m$ represents the operational coordinates of the UAV. 
then equalizing (3) and (4) an expression is obtained $\mathbf{A u}=\mathbf{b}$ :

$$
\underbrace{\mathbf{J}(\psi(k))}_{\mathbf{A}} \mathbf{v}(k)=\underbrace{\mathbf{v}_{\mathbf{d}}(k)+\frac{1}{T_{0}}\left(\mathbf{W}\left(\mathbf{P}_{\mathbf{d}}(k-1)-\mathbf{x}(k-1)\right)\right)}_{\mathbf{u}}
$$

Hence, the proposed control law is presented [18].

$$
\mathbf{v}_{\mathbf{c}}(k)=\mathbf{J}^{-\mathbf{1}}(\psi(k))\left(\mathbf{v}_{\mathbf{d}}(k)+\frac{1}{T_{0}}\left(\mathbf{W}\left(\mathbf{P}_{\mathbf{d}}(k-1)-\mathbf{x}(k-1)\right)\right)\right)
$$

\section{Stability Analysis}

This Section develops the stability analysis of the proposed control algorithm, The closed-loop equation is obtained by equating (5) with (3), considering $\mathbf{v}(k)=\mathbf{v}_{\mathbf{c}}(k)$

$$
\begin{aligned}
\frac{1}{T_{0}}(\mathbf{x}(k)-\mathbf{x}(k-1))= & \mathbf{J}(\psi(k)) \mathbf{J}^{-1}(\psi(k))\left(\mathbf{v}_{\mathbf{d}}(k)\right)+\ldots \\
& \frac{1}{T_{0}}(\mathbf{W} \underbrace{\left(\mathbf{P}_{\mathbf{d}}(k-1)-\mathbf{x}(k-1)\right)}_{\tilde{\mathbf{x}}(k-1)})
\end{aligned}
$$

considering: $\mathbf{J}(\psi(k)) \mathbf{J}^{-1}(\psi(k))=\mathbf{I}$, then reducing terms (6) can be rewritten as:

$$
\mathbf{x}(k)-\mathbf{x}(k-1)=T_{0} \mathbf{v}_{\mathbf{d}}(k)+\mathbf{W}(\tilde{\mathbf{x}}(k-1))
$$

Defined at the desired tracking velocity as, $\mathbf{v}_{\mathbf{d}}(k)=\frac{1}{T_{0}}\left(\mathbf{P}_{\mathbf{d}}(k)-\mathbf{P}_{\mathbf{d}}(k-1)-\Delta \gamma\right)$, then grouping equation the current states $(k)$ and the previous state $(k-1)$ there is

$$
\begin{gathered}
\underbrace{\mathbf{P}_{\mathbf{d}}(k-1)-\mathbf{x}(k-1)}_{\tilde{\mathbf{x}}(k-1)}=\underbrace{\mathbf{P}_{\mathbf{d}}(k)-\mathbf{x}(k)}_{\tilde{\mathbf{x}}(k)}-\Delta \gamma+\mathbf{W}(\tilde{\mathbf{x}}(k-1)) \\
\tilde{\mathbf{x}}(k-1)=\tilde{\mathbf{x}}(k)-\Delta \gamma+\mathbf{W}(\tilde{\mathbf{x}}(k-1)) \\
\Delta \gamma=\tilde{\mathbf{x}}(k)+\tilde{\mathbf{x}}(k-1)(\mathbf{W}-1)
\end{gathered}
$$

applied Z-transform to (7), the closed-loop equation is defined as:

$$
\begin{gathered}
\left(1-z^{-1}\right) \Delta \gamma(z)=\tilde{\mathbf{x}}(z)\left(1+z^{-1}(\mathbf{W}-1)\right) \\
\tilde{\mathbf{x}}(z)=\frac{\left(1-z^{-1}\right)}{\left(1+z^{-1}(\mathbf{W}-1)\right)} \Delta \gamma(z)
\end{gathered}
$$

where the poles of the system are defined as,

$$
1+z^{-1}(\mathbf{W}-1)=0
$$

so if $0<\mathbf{W}<1$ control errors $\tilde{\mathbf{x}}(k)=0$ when $k \rightarrow \infty$, in other words it's asymptotically stable.

\section{EXPERIMENTAL RESULTS}

This Section presents the different result's application of augmented reality for the assistance, maintenance and control of unmanned aerial vehicles. Fig.5 shows the structured environment, it has a monitor that shows augmented reality, the
Kinect camera for user tracking and the 3D Marker that will be detected by the camera for recognition in its virtualized form.

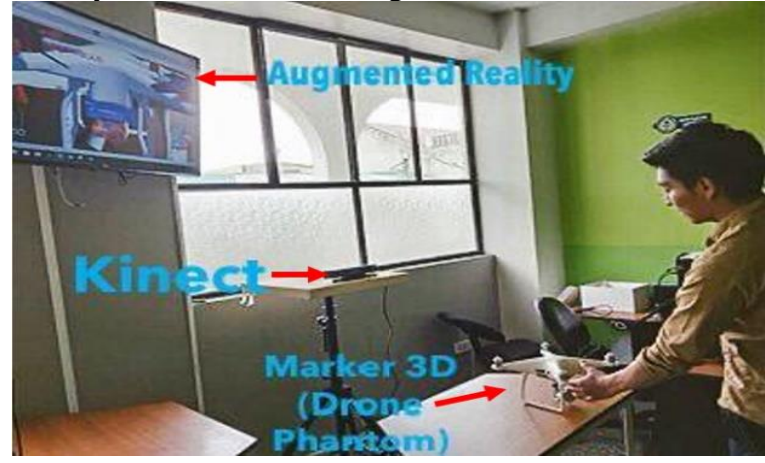

Figure 5. Operation Application

When the application is started the main menu appears with three scenes: Assistance, Maintenance and Control. The desired scene is accessed by clicking on the virtualized buttons with the user's hand as shown in Fig.2

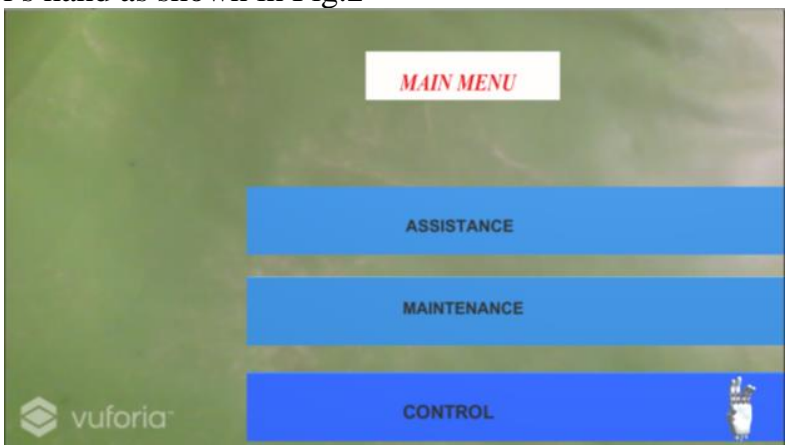

Figure 6. UAV Application Main Menu

A camera connected to the computer that visualizes the image or 3D object to be projected on the monitor, the recognition of $2 \mathrm{D}$ and $3 \mathrm{D}$ markers is making with the Vuforia application that allows to superimpose virtual objects to reality, in the Assistance Scene the scenes are divided according to the object that needs assistance(camera, propellers) as shown in Fig.7.

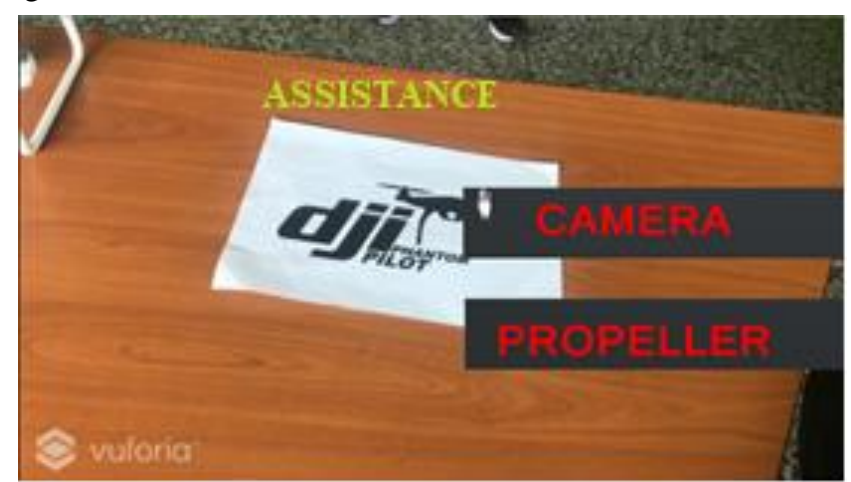

Figure 7. Assistance Menu

As shown in Fig.8 when entering the Camera Assistance scene this HMI interacts with the 2D marker, it depends on how the camera is focused on the 2D marker to have a more user friendly view. 


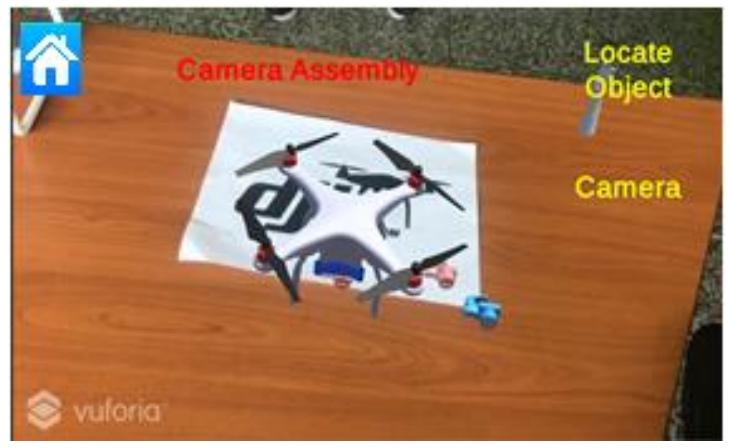

Figure 8. UAV Assistance Scene (Camera)

When the user closes his hand in the still camera position, the mobile camera appears and the user will move his hand in real time with the mobile camera as shown in the Fig.9.

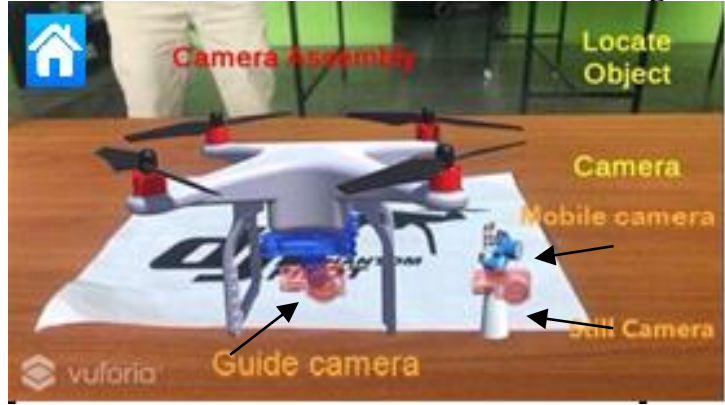

Figure 9. Camera Specification

The mobile camera should be fixed in the position of the guide camera until the message "Well placed" appears to ensure proper assistance as shown in the Fig. 10.

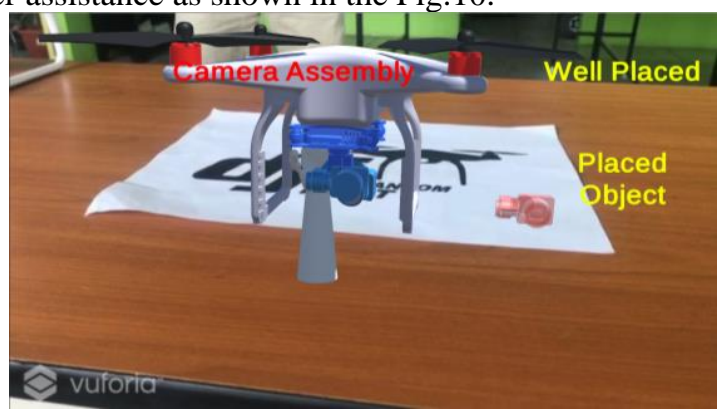

Figure 10. Finished Camera Assistance

Figure 11 shows the assistance scene for the propellers. Their operation is the same as that of the Fig.9 and Fig.10.

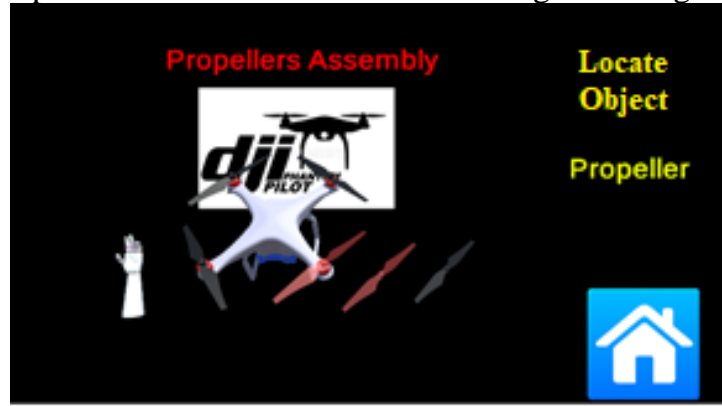

Figure 11. Assistance Scene (propellers)
When the maintenance scene is selected as shown in Fig.2 the HMI is immediately displayed, see Fig.12, this scene works in a real way with the 3D object by means of the virtualization showed by monitor. In the Maintenance scene the function is similar to the Assistance scene as shown in Fig.9 and Fig.10, it differs because the location of the objects (propellers and battery) is made considering the real position of the object in the UAV.

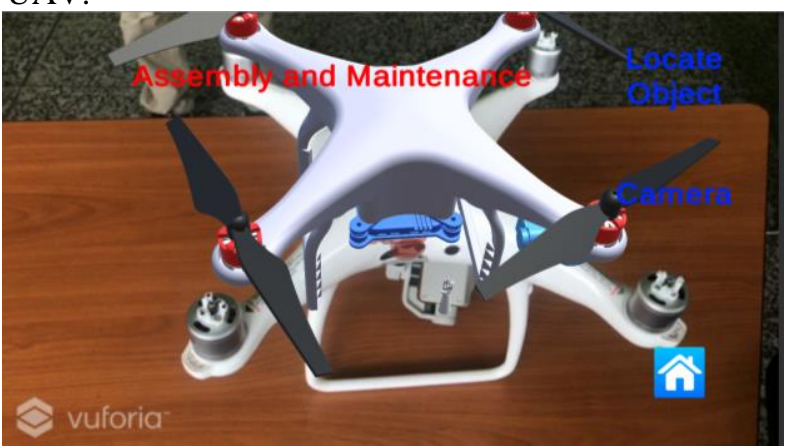

Figure 12. UAV Maintenance Scene

When selecting the control scene as shown in Fig.2 the HMI is accessed here is a virtual environment that allows the user to interact with this environment through his hand as shown in Fig.13. In this scene the HMI can be seen with the option to restart the scene, watch errors presented by the control and return to the main menu.

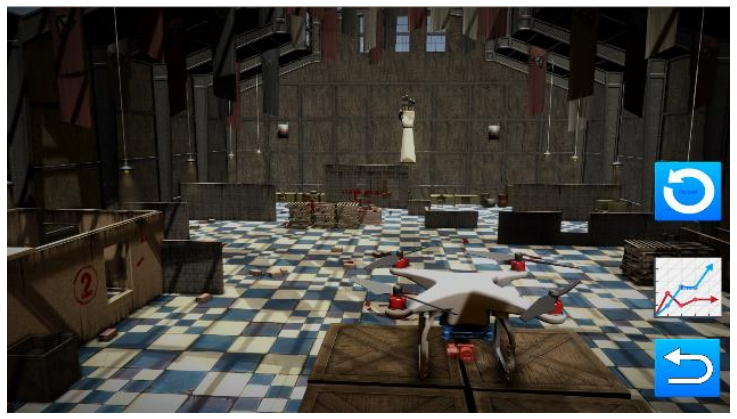

Figure 13. Control Scene

When the user closes his hand from this moment on, he begins to draw a route so that it becomes the gesture reference that the drone must follow as seen in the Fig.13.

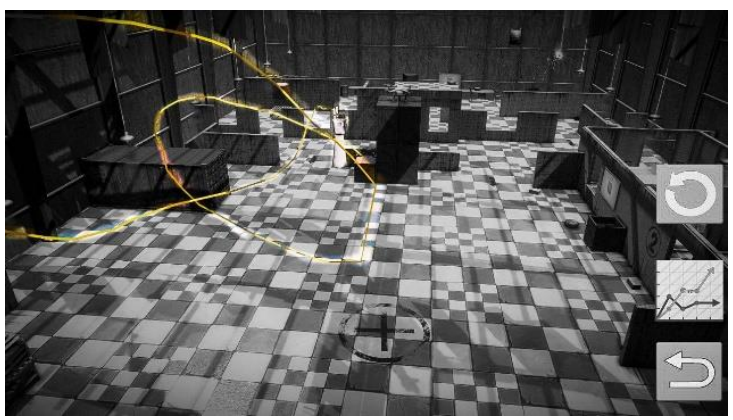

Figure 14. Traced Gesture Reference 
When the user finishes marking the desired path, the drone will begin to follow it by marking a desired path as shown in Fig.14

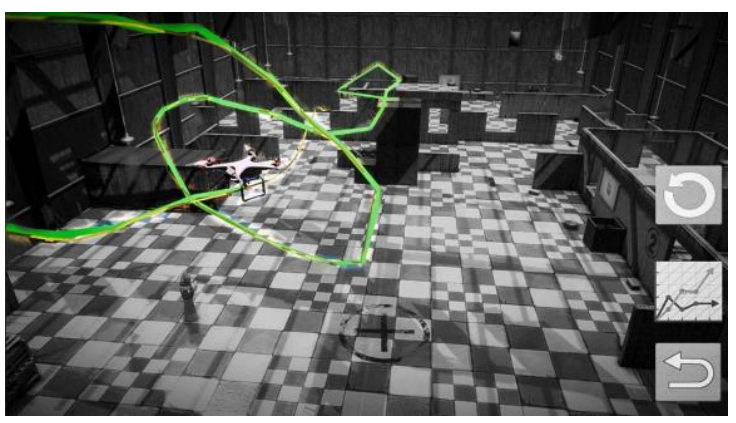

Figure 15. Desired vs. actual path

Once the desired path has been followed, the Drone will return to its initial position as shown in Fig.13. Clicking on the error button to view the graphs presented by the control, the scene is displayed as shown in the Fig. 16.

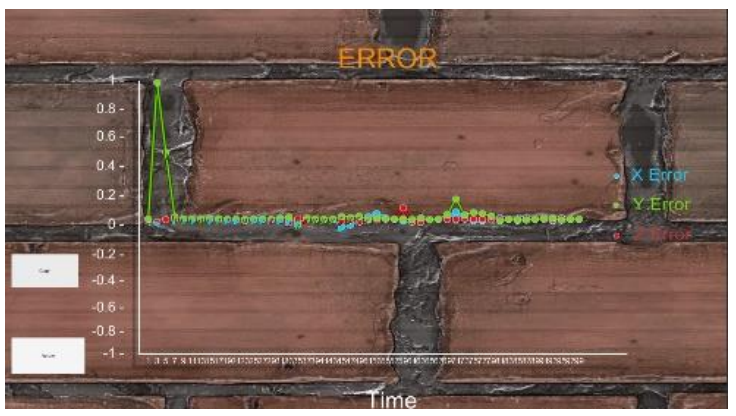

Figure 16. Control Errors Scene

\section{CONCLUSIONS}

In this article, a virtual and augmented reality environment was developed to assist in the assembly, maintenance and autonomous control of an unmanned aerial vehicle. The results obtained show the viability of including virtual and augmented reality systems in control processes that aim to minimize errors due to lack of knowledge about UAV assembly. The environment can be used as a means of training since it has the option of maintenance in a real UAV.

\section{ACKNOWLEDGMENT}

The authors would like to thank the Coorporación Ecuatoriana para el Desarrollo de la Investigación y Academia- CEDIA for their contribution in innovation, through the CEPRA projects, especially the project CEPRA-XIV-2020-08-RVA "Tecnologías Inmersivas MultiUsuario Orientadas a Sistemas Sinérgicos de Enseñanza-Aprendizaje "; also the Universidad de las Fuerzas Armadas ESPE and the Research Group ARSI, for the support for the development of this work.

\section{REFERENCES}

[1] Andaluz V.H., Castillo-Carrión D., Miranda R.J., Alulema J.: Virtual Reality Applied to Industrial Processes. In: De Paolis L., Bourdot P., Mongelli A. (eds) Aug-mented Reality, Virtual Reality, and Computer Graphics. AVR 2017. Lecture Notes in Computer Science, vol 10324, 5963 Springer, Cham (2017)
[2] Turner, C.J., Hutabarat, W., Oyekan, J., Tiwari, A.: Discrete event simulation and virtual reality use in industry: new opportunities and future trends. IEEE Trans. Hum.-Mach. Syst. 46, 882-894 (2016)

[3] Chicaiza E.A., De la Cruz E.I., Andaluz V.H.: Augmented Reality System for Training and Assistance in the Management of Industrial Equipment and Instruments. In: Bebis G. et al. (eds) Advances in Visual Computing. ISVC 2018. Lecture Notes in Computer Science, vol 11241, 676-680. Springer, Cham(2018)

[4] Ejder Bastug, Mehdi Bennis, Muriel Medard, Merouane Debbah.: Toward Intercon-nected Virtual Reality: Opportunities, Challenges, and Enablers. In: IEEE Communica-tions Magazine Volume: 55 , Issue: 6, 110-117, (June 2017 ).

[5] Tatić, D., Bojan, T.: The application of augmented reality technologies for the improvement of occupational safety in an industrial environment. Comput. Ind. 85, 1-10 (2017)

[6] Ortiz, J.S., Zapata, C.F., Vega, A.D., Andaluz, V.H.: Path planning based on visual feedback between terrestrial and aerial robots cooperation. In: Zeghloul, S., Romdhane, L., Laribi, M. A. (eds.) Computational Kinematics. MMS, vol. 50, pp. 96-105. Springer, Cham (2018).

[7] Andaluz, Víctor H., et al.: Nonlinear controller of quadcopters for agricultural monitoring. In: Bebis, G., et al. (eds.) ISVC 2015. LNCS, vol. 9474, pp. 476-487. Springer, Cham (2015).

[8] Pusda F.R., Valencia F.F., Andaluz V.H., Zambrano V.D. (2019) Training Assistant for Automotive Engineering Through Augmented Paolis L., Bourdot P. (eds) Augmented Reality, Virtual Reality, and Computer Graphics. AVR 2019

[9] Rios H., Hincapié M., Caponio A., Mercado E., González Mendívil E. (2011) Aug-mented Reality: An Advantageous Option for Complex Training and Maintenance Op-erations in Aeronautic Related Processes. In: Shumaker R. (eds) Virtual and Mixed Re-ality - New Trends. VMR 2011.

[10] Guerrero, B. \& Valero, L.: Efectos secundarios de la realidad virtual inmersiva. In: International Journal of Psychology and Psychological Therapy, 13 (2), 163-178. (2013).

[11] Katharina Pentenrieder, Christian Bade, Fabian Doil, Peter Meier: Augmented Reality-based factory planning - an application tailored to industrial needs. In: 2007 6th IEEE and ACM International Symposium on Mixed and Augmented Reality, 31-41, Nara, (2007).

[12] Andaluz V.H. et al.: Transparency of a Bilateral Tele-Operation Scheme of a Mobile Manipulator Robot. In: De Paolis L., Mongelli A. (eds) Augmented Reality, Vir-tual Reality, and Computer Graphics. AVR 2016. Lecture Notes in Computer Science, vol 9768, 228-245. Springer, Cham (2016).

[13] Michalos G, Karagiannis P, Makris S, Tokc,alar O., Chryssolouris G Augmented Reality (AR) Applications for Supporting Human-Robot Interactive Cooperation. In:48th CIRP Conference on Manufacturing Systems. CIRP-1447 1-4, Naples, Italy Procedia (2015)

[14] Andaluz, V.H., Carvajal, C.P., Pérez, J.A., Proaño, L.E.: Kinematic nonlinear control of aerial mobile manipulators. In: Huang, Y., Wu, H., Liu, H., Yin, Z. (eds.) ICIRA 2017.LNCS (LNAI), vol. 10464, pp. 740749. Springer, Cham (2017).

[15] Romero J., Quero, et al.: Training Assistant for Industrial Processes through Augmented Reality. In: ICETC 2019: Proceedings of the 2019 11th International Conference on Education Technology and Computers, 308-315. (October 2019). https://doi.org/10.1145/3369255.3369295

[16] Macmahon N. Merino O., Maza C, Jimenez a., Delgado A.: Simulador de Ensamble de Dispositivos Electrónicos por medio de Realidad Aumentada usando un SensorKinect. In: Pistas Educativas, No. 108, Vol. 35, 1436-1449, (Octubre 2014).

[17] Acosta Julio, Andaluz Victor, González-de-Rivera G.; Garrido J.: EnergySaver Mobile Manipulator Based on Numerical Methods. Electronics 8, 1100. (2019)

[18] Andaluz V.H. et al. Autonomous Monitoring of Air Quality Through an Unmanned Aerial Vehicle. In: Wotawa F., Friedrich G., Pill I., KoitzHristov R., Ali M. (eds) Advances and Trends in Artificial Intelligence. From Theory to Practice. IEA/AIE 2019. Lecture Notes in Computer Science, vol 11606, 148-150. Springer, Cham (2019) 
